\title{
PROGÊNIES DE COFFEA ARABICA CV IPR-100 RESISTENTES AO NEMATÓIDE MELOIDOGYNE PARANAENSIS ( $\left.{ }^{1}\right)$
}

\author{
GUSTAVO HIROSHI SERA $\left(\left(^{*}, 6\right)\right.$; TUMORU SERA $\left({ }^{3}\right)$; DHALTON SHIGUER ITO $\left({ }^{4}\right)$; \\ JOÃO SIQUEIRA DA MATA $\left({ }^{4}\right)$; DEISY SAORI DOI $\left({ }^{5,6}\right)$; JOSÉ ALVES DE AZEVEDO $\left({ }^{3}\right)$; \\ CLAUDIONOR RIBEIRO FILHO $\left({ }^{4}\right)$
}

\begin{abstract}
RESUMO
O objetivo deste trabalho foi avaliar o nível de resistência a Meloidogyne paranaensis em progênies de cafeeiros (Coffea arabica) de frutos maiores da mesma família da cultivar IPR-100. O experimento de 64 tratamentos foi desenvolvido em casa de vegetação no delineamento em blocos ao acaso com 3 repetições e parcelas com 20 plântulas. A cultivar Mundo Novo IAC 376-4 foi utilizada como testemunha suscetível a M. paranaensis. Foram inoculados 500 ovos por planta, totalizando 10000 ovos por parcela de $100 \mathrm{~cm}^{2}$. Avaliou-se, 109 dias após as inoculações, o número de galhas e a massa de ovos presentes nas raízes. As plantas-mãe das 24 progênies classificadas como resistentes ao $M$. paranaensis têm potencial para se tornarem a versão melhorada da cultivar IPR-100 com frutos maiores.
\end{abstract}

Palavras-chave: Coffea arabica, melhoramento, cultura do café, nematóide das galhas, resistência.

\section{ABSTRACT \\ PROGENIES OF COFFEA ARABICA CV IPR-100 RESISTANT TO ROOT-KNOT NEMATODE MELOIDOGYNE PARANAENSIS}

The aim of this research was to identify large grain progenies of Coffea arabica cV IPR-100 with various level resistance to Meloidogyne paranaensis. The experiment was performed in greenhouse by artificial inoculation, with a experimental design of randomized blocks with 64 treatments (63 C. arabica 'IPR-100' progenies + C. arabica 'Mundo Novo'), 3 replications and 20 plants per plot. The Mundo Novo IAC 376-4 cultivar was used as susceptible standard to $M$. paranaensis. Five hundred eggs per plant were inoculated, totalizing 10000 eggs per plot of $100 \mathrm{~cm}^{2}$. Gall and egg mass indexes were evaluated 109 days after the inoculations. The mother plants of the 24 progenies classified as resistants to M. paranaensis have potential to become an improved version of 'IPR-100' with large grains.

Key-words: Coffea arabica, breeding, coffee crop, root-knot nematode, resistance.

$\left({ }^{1}\right)$ Trabalho parcialmente financiado pelo Consórcio Brasileiro de P\&D Café. Recebido para publicação em 7 de junho de 2005 e aceito em 15 de agosto de 2006.

$\left({ }^{2}\right)$ Mestrando em Genética da Universidade Estadual de Londrina (UEL). E-mails: gustavosera@uol.com.br * Autor correspondente; tsera@uol.com.br; tsera@iapar.br

$\left({ }^{3}\right)$ Instituto Agronômico do Paraná (IAPAR), Caixa Postal 481, 86001-970 Londrina (PR).

$\left({ }^{4}\right)$ Bolsistas da FUNAPE / Embrapa / Consórcio Brasileiro de P \& D Café - Núcleo de Genética.

$\left({ }^{5}\right)$ Acadêmica do curso de Agronomia da UEL, Londrina (PR).

$\left({ }^{6}\right)$ Bolsista do CNPq. 


\section{INTRODUÇÃO}

Na cafeicultura brasileira houve grandes prejuízos econômicos devido à ocorrência de nematóides do gênero Meloidogyne. A redução estimada da produção mundial de café causada pelos fitonematóides é de 15\% segundo SASSER (1979). Conforme Lordello (1976, citado por GonçALVES et al., 2004) a redução da produção brasileira de café é estimada em cerca de $20 \%$, e desse total, as espécies de Meloidogyne são responsáveis por $75 \%$. GonçALves et al. (2004) ainda relataram que é necessário considerar as perdas indiretas causadas pelo parasitismo dos nematóides como a menor tolerância ao frio e à seca e a perda parcial na eficiência de utilização de alguns insumos.

Atualmente, existem quatorze espécies de Meloidogyne que parasitam o cafeeiro das quais seis ocorrem no Brasil (M. paranaensis, M. incognita, $M$. exigua, M. coffeicola, M. goeldii e M. hapla), segundo SANTOS (2001). As mais prejudiciais são M. exigua, pela ampla distribuição geográfica, e $M$. paranaensis e $M$. incognita pela intensidade dos danos que causam (GonÇALVES et al., 2004). Pesquisa recente realizada por Carneiro e Almeida (2000) indica um substancial aumento da distribuição de $M$. paranaensis $(70 \%)$ e decréscimo de M. incognita (30 \%) no Paraná. Em cafezais paulistas, $M$. paranaensis está disseminado em freqüências que variam de $10,7 \%$ a $24,5 \%$ das amostras em que foram encontrados nematóides do gênero Meloidogyne (LORDELLO e LORDELLO, 2001), enquanto em Minas Gerais, existe apenas um relato de sua ocorrência (SANTOS, 1997).

$\mathrm{Na}$ maioria das vezes, o controle de fitonematóides em cafezais é ineficiente e se a área estiver contaminada é praticamente impossível eliminá-los (Gonçalves e Silvarolla, 2001). As estratégias de manejo para se diminuir a população de nematóides são: cultural, biológico, químico e genético (GONÇALVES et al., 1998), sendo o último o mais eficiente e viável economicamente. Entretanto, conforme GonçALVES et al. (1998), a principal estratégia de manejo ainda é evitar a disseminação de fitonematóides por solos, águas e culturas.

A resistência ao $M$. incognita e $M$. paranaensis vem sendo encontrada em C. canephora (GONÇALVES et al., 1988; 1996; Sera et al., 2004b; 2005) e em C. congensis (GONÇALVES et al., 1988).

Fontes de resistência a $M$. paranaensis (MatA et al., 2000, 2002; SERA et al., 2002, 2004a) e a M. incognita (CARNeIro, 1995; MATA et al., 2002; SERA et al., 2004a) têm sido constatadas em plantas do Icatu (C. arabica). Resistência para algumas raças de $M$. incognita foram também identificadas em cafeeiros arábicos do germoplasma Sarchimor (GonçALVES et al., 1988). Gonçalves e Silvarolla (2001) relataram que os germoplasmas $C$. arabica $\times C$. canephora, Icatu, Sarchimor e Catimor são resistentes a M. exigua, $M$. incognita e M. paranaensis, porém, geralmente, segregantes para essa característica. As cultivares IPR 100 e IPR 106 são moderadamente resistentes a $M$. paranaensis em homozigoze, de acordo com SERA et al. (2002).

Mata et al. (2000) identificaram em área altamente infestada com M. paranaensis um genótipo de Catucaí (IAPAR Vit. 83), o qual deu origem à cultivar IPR-100, com $100 \%$ das plantas resistentes. Nesse genótipo, observaram-se produção e vigor vegetativo normais nessa área infestada, enquanto em outros genótipos desse mesmo experimento ocorreram baixíssima produção e vigor vegetativo, além de muitas plantas mortas. Progênie de uma das plantas desse genótipo com frutos grandes (IAPAR Vit. 83-5) está em observação em um campo experimental do IAPAR, no Paraná.

O objetivo deste trabalho foi identificar plantas com resistência ao $M$. paranaensis em cafeeiros de frutos grandes na progênie original (IAPAR Vit. 83-5) da cultivar IPR 100.

\section{MATERIAL E MÉTODOS}

O experimento em casa de vegetação foi instalado em 5 de novembro de 2003, no Instituto Agronômico do Paraná (IAPAR), no município de Londrina.

Foram avaliadas 63 progênies do germoplasma IAPAR Vit. 83-5, cujos frutos são maiores do que os da cultivar Catuaí Vermelho IAC 81. A cultivar Mundo Novo IAC 376-4 foi utilizada como testemunha suscetível.

As sementes foram germinadas em areia e repicadas no estádio "palito de fósforo" para uma caixa de cimento amianto de 500 litros, reproduzindo as condições de campo usando solo arenoso esterilizado, irrigado e adubado de acordo com a necessidade. Foi usado o delineamento experimental em blocos ao acaso, com três repetições e parcelas de 20 plantas. As plantas foram repicadas com distâncias entre linha e entre planta de, respectivamente, $10 \mathrm{~cm}$ e $0,5 \mathrm{~cm}$, totalizando, $100 \mathrm{~cm}^{2}$ de área por parcela.

Os inóculos iniciais utilizados foram provenientes de solo e raízes de cafeeiros suscetíveis, em áreas naturalmente infestadas 
por M. paranaensis, identificadas por KRZYZANOWSKI et al. (2001). Foram utilizadas plantas diferenciadoras para confirmar que a inoculação estava sendo realizada com $M$. paranaensis (CARneIro e Almeida, 2000). Posteriormente, o inóculo foi multiplicado em cafeeiros. Para o preparo do inóculo, utilizouse a técnica de obtenção de ovos e juvenis através do método proposto por TAYLOR e SASSER (1978).

Foram realizadas três inoculações, 43 dias após a semeadura, distribuindo 500 ovos ao redor de cada planta, totalizando em torno de 10.000 ovos por parcela de $100 \mathrm{~cm}^{2}$. As três inoculações foram feitas seqüencialmente visando diminuir os erros na quantidade de ovos inoculados. A avaliação nematológica para triagens iniciais, descrita por FAzUOLI et al. (1984), foi realizada 109 dias após as inoculações, através da contagem de galhas e massas de ovos (GO), após coloração das raízes com floxina B. Utilizou-se a escala de TAYLOR (1971) adaptada, sendo utilizadas notas de 1 a 6 , sendo nota $1=$ ausência de GO; nota $2=1$ a 2 GO; nota $3=$ de 3 a $10 \mathrm{GO}$; nota $4=$ de 11 a $30 \mathrm{GO}$; nota $5=31$ a $100 \mathrm{GO}$; nota $6=$ mais de $100 \mathrm{GO}$.

Foi utilizado o programa estatístico Genes (CRUZ, 2001) para análise de variância ao nível de média da parcela e comparar as médias pelo teste ScottKnott a 1\% de significância. Antes de realizar a análise de variância, foi feito o teste de Cochran para se verificar a homogeneidade das variâncias (G máximo) a $1 \%$.

Foram consideradas plantas resistentes aquelas com notas 1, 2 e 3 e suscetíveis como 4, 5 e 6, com base no critério modificado de SASSER et al. (1984), que classificaram plantas como resistentes aquelas com número de galhas menor ou igual a dez e, as com valores superiores foram consideradas suscetíveis.

\section{RESULTADOS E DISCUSSÃO}

O teste do G máximo indicou homogeneidade das variâncias obtidas. Pela análise de variância pelo teste $\mathrm{F}$ a $1 \%$ observou-se que existem diferenças significativas entre os tratamentos (Tabela 1). O nível de precisão experimental foi satisfatório com coeficiente de variação de 10,15\%. O coeficiente de determinação genotípica máxima foi de 86,66\%, concluindo ser relativamente fácil selecionar para a resistência a Meloidogyne paranaensis.

$\mathrm{Na}$ tabela 2, observam-se o índice médio de galhas e/ ou massas de ovos (IGO), a porcentagem de plantas resistentes a $M$. paranaensis e o grau de resistência dos genótipos avaliados. A freqüência de plantas segundo o índice de galhas e/ ou massas de ovos é apresentada na tabela 3.

Tabela 1. Análise de variância da variável índice de galhas e/ou massas de ovos de $M$. paranaensis, em progênies de cafeeiros da cultivar IPR-100, com inoculação artificial em casa de vegetação

\begin{tabular}{lcccc}
\hline F.V. & G. L. & S. Q. & Q. M. & F \\
\hline Blocos & 2 & 0,700716 & 0,350358 & \\
Tratamentos & 63 & 42,559538 & 0,675548 & $7,496^{* *}$ \\
Resíduo & 126 & 11,3553 & 0,090121 & \\
\hline Total & 191 & 54,615554 & & \\
\hline
\end{tabular}

** significativo pelo teste F a $1 \%$ de probabilidade.

Pelo teste de médias foi possível classificar as progênies em quatro grupos de resistência $(a, b, c, d)$, diferentes estatisticamente a $1 \%$ de probabilidade, sendo 11 delas consideradas suscetíveis (a), 13 como moderadamente suscetíveis (b), 16 como moderadamente resistentes (c) e 24 como resistentes (d). A testemunha 'Mundo Novo IAC 376-4' foi classificada como suscetível. A nota média do índice de galhas e/ ou massas de ovos (IGO) desses genótipos considerados resistentes foi de 2,50, em comparação com a testemunha suscetível ('Mundo Novo IAC 376-4') com nota média de 4,18. Das 63 progênies da cultivar IPR-100, 31 tiveram porcentagem de plantas resistentes menor do que $90 \%, 23$ com $90 \%$ a $94 \%$ de plantas resistentes, oito com $95 \%$ a $99 \%$ e somente a progênie IAPAR 83-5/ 6-6 teve 100\% de plantas resistentes. (Tabela 2). As poucas plantas suscetíveis dos genótipos resistentes são de nota 4 para o índice de galhas e massas de ovos, enquanto na testemunha suscetível Mundo Novo predominam as notas 4 e 5 , sendo um dos poucos genótipos de plantas com nota 6 (Tabela 3).

Como se observou no padrão suscetível Mundo Novo 20,8 \% de plantas com nota 3 na escala de pontos adotada, é possível que apesar da alta freqüência de plantas resistentes em algumas progênies, esses genótipos sejam segregantes para a resistência.

Pelo baixo IGO, alta freqüência de plantas resistentes e a predominância de plantas com notas 1, 2 e 3 de IGO nos genótipos classificados como resistentes, foi possível confirmar a resistência ao $M$. paranaensis do genótipo IAPAR Vit. 83-5, em condições de campo, em uma área altamente infestada por este nematóide segundo MATA et al. (2000). 
Tabela 2. Teste de médias da variável índice de galhas e/ ou massas de ovos do nematóide M. paranaensis (IGO), porcentagem de plantas resistentes (\% Res) e graus de resistência (GR) de 63 progênies da cultivar IPR 100 (Coffea arabica) e da testemunha suscetível 'Mundo Novo IAC 376-4' (IAPAR, Londrina, PR)

\begin{tabular}{|c|c|c|c|c|c|c|c|c|c|}
\hline Trat. & Genótipos & $\operatorname{IGO}\left({ }^{1}\right)$ & Res. $\left({ }^{2}\right)$ & GR $\left(^{3}\right)$ & Trat & Genótipos & $\operatorname{IGO}\left({ }^{1}\right)$ & $\operatorname{Res}\left({ }^{2}\right)$ & $\operatorname{GR}\left({ }^{3}\right)$ \\
\hline & & & $\%$ & & & & & $\%$ & \\
\hline 64 & M. Novo & $4,18 \mathrm{a}$ & 20,8 & $S$ & 27 & IAPAR 83-5 / 4-14 & $2,85 \mathrm{c}$ & 81,7 & MR \\
\hline 37 & IAPAR 83-5 / 5-13 & $4,12 \mathrm{a}$ & 17,6 & $S$ & 46 & IAPAR 83-5 / 6-5 & $2,79 \mathrm{c}$ & 87,8 & MR \\
\hline 55 & IAPAR 83-5 / 6-14 & $3,75 \mathrm{a}$ & 36,8 & $S$ & 34 & IAPAR 83-5 / 5-9 & $2,75 \mathrm{c}$ & 93,2 & MR \\
\hline 28 & IAPAR 83-5 / 5-1 & $3,74 \mathrm{a}$ & 44,5 & $S$ & 58 & IAPAR 83-5 / 6-18 & $2,74 \mathrm{c}$ & 92,5 & MR \\
\hline 1 & IAPAR 83-5 / 1-1 & $3,69 \mathrm{a}$ & 46,4 & $S$ & 26 & IAPAR 83-5 / 4-12 & $2,73 \mathrm{c}$ & 91,8 & MR \\
\hline 15 & IAPAR 83-5 / 3-7 & $3,68 \mathrm{a}$ & 45,6 & $S$ & 14 & IAPAR 83-5 / 3-6 & $2,73 \mathrm{c}$ & 84,5 & MR \\
\hline 33 & IAPAR 83-5 / 5-7 & $3,63 \mathrm{a}$ & 45,2 & $S$ & 56 & IAPAR 83-5 / 6-15 & $2,72 \mathrm{c}$ & 93,3 & MR \\
\hline 19 & IAPAR 83-5 / 4-3 & $3,60 \mathrm{a}$ & 46,7 & $S$ & 12 & IAPAR 83-5 / 3-4 & $2,69 \mathrm{c}$ & 91,5 & MR \\
\hline 11 & IAPAR 83-5 / 3-3 & $3,57 \mathrm{a}$ & 48,2 & $S$ & 54 & IAPAR 83-5 / 6-13 & $2,65 \mathrm{~d}$ & 92,3 & $\mathrm{R}$ \\
\hline 61 & IAPAR 83-5 / 6-22 & $3,55 \mathrm{a}$ & 55,8 & $S$ & 59 & IAPAR 83-5 / 6-19 & $2,65 \mathrm{~d}$ & 92,3 & $\mathrm{R}$ \\
\hline 38 & IAPAR 83-5 / 5-14 & $3,52 \mathrm{a}$ & 57,9 & $S$ & 52 & IAPAR 83-5 / 6-11 & $2,63 \mathrm{~d}$ & 91,1 & $\mathrm{R}$ \\
\hline 49 & IAPAR 83-5 / 6-8 & $3,46 \mathrm{~b}$ & 55,5 & MS & 32 & IAPAR 83-5 / 5-6 & $2,62 \mathrm{~d}$ & 91,4 & $\mathrm{R}$ \\
\hline 7 & IAPAR 83-5 / 2-5 & $3,44 \mathrm{~b}$ & 54,3 & MS & 9 & IAPAR 83-5 / 3-1 & $2,61 \mathrm{~d}$ & 92,2 & $\mathrm{R}$ \\
\hline 21 & IAPAR 83-5 / 4-5 & $3,36 \mathrm{~b}$ & 65,8 & MS & 39 & IAPAR 83-5 / 5-16 & $2,60 \mathrm{~d}$ & 93,2 & $\mathrm{R}$ \\
\hline 13 & IAPAR 83-5 / 3-5 & $3,36 \mathrm{~b}$ & 56,8 & MS & 8 & IAPAR 83-5 / 2-6 & $2,59 \mathrm{~d}$ & 91,5 & $\mathrm{R}$ \\
\hline 18 & IAPAR 83-5 / 4-2 & $3,35 \mathrm{~b}$ & 59,5 & MS & 20 & IAPAR 83-5 / 4-4 & $2,59 \mathrm{~d}$ & 90,6 & $\mathrm{R}$ \\
\hline 22 & IAPAR 83-5 / 4-6 & $3,31 \mathrm{~b}$ & 59,2 & MS & 48 & IAPAR 83-5 / 6-7 & $2,56 \mathrm{~d}$ & 93,9 & $\mathrm{R}$ \\
\hline 16 & IAPAR 83-5 / 3-8 & $3,29 \mathrm{~b}$ & 66,7 & MS & 44 & IAPAR 83-5 / 6-3 & $2,55 \mathrm{~d}$ & 93,3 & $\mathrm{R}$ \\
\hline 25 & IAPAR 83-5 / 4-9 & $3,27 \mathrm{~b}$ & 75,9 & MS & 62 & IAPAR 83-5 / 6-23 & $2,55 \mathrm{~d}$ & 93,3 & $\mathrm{R}$ \\
\hline 29 & IAPAR 83-5 / 5-2 & $3,24 \mathrm{~b}$ & 76,5 & MS & 4 & IAPAR 83-5 / 2-2 & $2,54 \mathrm{~d}$ & 96,4 & $\mathrm{R}$ \\
\hline 31 & IAPAR 83-5 / 5-5 & $3,23 \mathrm{~b}$ & 73,0 & MS & 10 & IAPAR 83-5 / 3-2 & $2,53 \mathrm{~d}$ & 88,3 & $\mathrm{R}$ \\
\hline 5 & IAPAR 83-5 / 2-3 & $3,17 \mathrm{~b}$ & 64,3 & MS & 41 & IAPAR 83-5 / 5-18 & $2,53 \mathrm{~d}$ & 98,1 & $\mathrm{R}$ \\
\hline 60 & IAPAR 83-5 / 6-20 & $3,11 \mathrm{~b}$ & 81,2 & MS & 51 & IAPAR 83-5 / 6-10 & $2,52 \mathrm{~d}$ & 96,6 & $\mathrm{R}$ \\
\hline 53 & IAPAR 83-5 / 6-12 & $3,11 \mathrm{~b}$ & 80,0 & MS & 40 & IAPAR 83-5 / 5-17 & $2,51 \mathrm{~d}$ & 95,6 & $\mathrm{R}$ \\
\hline 24 & IAPAR 83-5 / 4-8 & $3,05 \mathrm{c}$ & 74,4 & MR & 30 & IAPAR 83-5 / 5-4 & $2,49 \mathrm{~d}$ & 90,9 & $\mathrm{R}$ \\
\hline 35 & IAPAR 83-5 / 5-10 & $3,04 \mathrm{c}$ & 91,3 & MR & 17 & IAPAR 83-5 / 4-1 & $2,44 \mathrm{~d}$ & 94,2 & $\mathrm{R}$ \\
\hline 6 & IAPAR 83-5 / 2-4 & $2,98 \mathrm{c}$ & 75,4 & MR & 57 & IAPAR 83-5 / 6-16 & $2,43 \mathrm{~d}$ & 92,7 & $\mathrm{R}$ \\
\hline 23 & IAPAR 83-5 / 4-7 & $2,93 \mathrm{c}$ & 90,0 & MR & 47 & IAPAR 83-5 / 6-6 & $2,43 \mathrm{~d}$ & 100,0 & $\mathrm{R}$ \\
\hline 50 & IAPAR 83-5 / 6-9 & $2,92 \mathrm{c}$ & 72,1 & MR & 45 & IAPAR 83-5 / 6-4 & $2,40 \mathrm{~d}$ & 96,2 & $\mathrm{R}$ \\
\hline 63 & IAPAR 83-5 / 6-24 & $2,91 \mathrm{c}$ & 81,2 & MR & 42 & IAPAR 83-5 / 6-1 & $2,39 \mathrm{~d}$ & 95,6 & $\mathrm{R}$ \\
\hline 36 & IAPAR 83-5 / 5-12 & $2,90 \mathrm{c}$ & 92,1 & MR & 3 & IAPAR 83-5 / 1-3 & $2,32 \mathrm{~d}$ & 96,6 & $\mathrm{R}$ \\
\hline 43 & IAPAR 83-5 / 6-2 & $2,89 \mathrm{c}$ & 90,0 & MR & 2 & IAPAR 83-5 / 1-2 & $1,89 \mathrm{~d}$ & 94,8 & $\mathrm{R}$ \\
\hline
\end{tabular}

$\left({ }^{1}\right)$ Médias seguidas pelas mesmas letras não diferem estatisticamente entre si pelo teste Scott-Knott, a $1 \%$. Escala do índice de galhas e/ ou massas de ovos: 1 = 0 galhas e/ ou massas de ovos (GO); $2=1$ a 2 GO; $3=3$ a 10 GO; $4=11$ a 30 GO; $5=31$ a 100 GO; $6=$ mais de 100 GO.

${ }^{(2)}$ Plantas com notas 1, 2 e 3 de IGO são resistentes e plantas com notas 4, 5 e 6 são suscetíveis.

( $\left.{ }^{3}\right) \mathrm{S}$ = suscetível; MS = moderadamente suscetível; $\mathrm{MR}=$ moderadamente resistente; $\mathrm{R}=$ resistente. 
Tabela 3. Freqüência de plantas (\%) segundo o índice de galhas e/ ou massas de ovos (IGO) das progênies da cultivar IPR-100 (IAPAR 83-5) e do padrão suscetível 'Mundo Novo IAC 376-4' (M. N.) avaliados em Londrina, PR, para a resistência ao nematóide $M$. paranaensis

\begin{tabular}{|c|c|c|c|c|c|c|c|c|c|c|c|c|c|}
\hline \multirow{2}{*}{ Trat $\left({ }^{1}\right)$} & \multicolumn{3}{|c|}{ Freqüência de plantas } & \multicolumn{3}{|c|}{ segundo o IGO $\left({ }^{2}\right)$} & \multirow{2}{*}{ Trat $\left({ }^{1}\right)$} & \multicolumn{6}{|c|}{ Freqüência de plantas segundo o IGO $\left({ }^{2}\right)$} \\
\hline & 1 & 2 & 3 & 4 & 5 & 6 & & 1 & 2 & 3 & 4 & 5 & 6 \\
\hline & \multicolumn{6}{|c|}{$\%$} & & \multicolumn{6}{|c|}{$\%$} \\
\hline M. N. & -- & -- & 20,8 & 47,0 & 26,2 & 6,0 & 27 & 6,7 & 23,3 & 51,7 & 15,0 & 3,3 & -- \\
\hline 37 & -- & -- & 17,6 & 58,8 & 23,5 & -- & 46 & -- & 38,8 & 49,0 & 10,2 & 2,0 & -- \\
\hline 55 & -- & -- & 36,8 & 52,7 & 10,5 & -- & 34 & 6,8 & 18,6 & 67,8 & 6,8 & -- & -- \\
\hline 28 & -- & 5,6 & 38,9 & 33,3 & 20,4 & 1,8 & 58 & -- & 30,0 & 62,5 & 7,5 & -- & -- \\
\hline 1 & -- & 10,7 & 35,7 & 30,4 & 23,2 & -- & 26 & -- & 36,7 & 55,1 & 8,2 & -- & -- \\
\hline 15 & -- & 4,3 & 41,3 & 34,8 & 19,6 & -- & 14 & 5,2 & 34,5 & 44,8 & 12,1 & 3,4 & -- \\
\hline 33 & -- & 9,4 & 35,8 & 37,8 & 17,0 & -- & 56 & -- & 33,3 & 60,0 & 6,7 & -- & -- \\
\hline 19 & -- & -- & 46,7 & 46,8 & 6,7 & -- & 12 & -- & 46,8 & 44,7 & 8,5 & -- & -- \\
\hline 11 & -- & 10,7 & 37,5 & 28,6 & 21,4 & 1,8 & 54 & -- & 42,3 & 50,0 & 7,7 & -- & -- \\
\hline 61 & -- & -- & 55,8 & 34,6 & 9,6 & -- & 59 & -- & 42,3 & 50,0 & 7,7 & -- & -- \\
\hline 38 & -- & -- & 57,9 & 31,6 & 10,5 & - & 52 & -- & 39,3 & 51,8 & 8,9 & -- & -- \\
\hline 49 & -- & 4,4 & 51,1 & 35,6 & 8,9 & -- & 32 & -- & 46,6 & 44,8 & 8,6 & -- & -- \\
\hline 7 & -- & 20,0 & 34,3 & 31,4 & 14,3 & -- & 9 & -- & 45,1 & 47,1 & 7,8 & -- & -- \\
\hline 21 & -- & 2,4 & 63,4 & 29,3 & 4,9 & -- & 39 & 16,9 & 13,6 & 62,7 & 6,8 & -- & -- \\
\hline 13 & -- & 13,7 & 43,1 & 37,3 & 5,9 & -- & 8 & 3,4 & 42,4 & 45,7 & 8,5 & -- & -- \\
\hline 18 & -- & 11,9 & 47,6 & 38,1 & 2,4 & -- & 20 & -- & 51,0 & 39,6 & 9,4 & -- & -- \\
\hline 22 & -- & 14,3 & 44,9 & 28,6 & 12,2 & -- & 48 & -- & 53,1 & 40,8 & 6,1 & -- & -- \\
\hline 16 & -- & 5,6 & 61,1 & 33,3 & -- & -- & 44 & -- & 51,1 & 42,2 & 6,7 & -- & -- \\
\hline 25 & -- & -- & 75,9 & 20,7 & 3,4 & -- & 62 & -- & 51,1 & 42,2 & 6,7 & -- & -- \\
\hline 29 & -- & -- & 76,5 & 23,5 & -- & -- & 4 & -- & 49,1 & 47,3 & 3,6 & -- & -- \\
\hline 31 & -- & 13,5 & 59,5 & 24,3 & 2,7 & -- & 10 & 5,0 & 48,3 & 35,0 & 11,7 & -- & -- \\
\hline 5 & 10,7 & 7,2 & 46,4 & 28,6 & 7,1 & - & 41 & -- & 50,0 & 48,1 & 1,9 & -- & -- \\
\hline 60 & -- & 14,6 & 66,6 & 14,6 & 4,2 & -- & 51 & 8,6 & 34,6 & 53,4 & 3,4 & -- & -- \\
\hline 53 & -- & 17,1 & 62,9 & 17,1 & 2,9 & -- & 40 & 4,4 & 44,5 & 46,7 & 4,4 & -- & -- \\
\hline 24 & -- & 4,7 & 69,7 & 25,6 & -- & -- & 30 & 10,9 & 30,9 & 49,1 & 9,1 & -- & -- \\
\hline 35 & -- & 4,3 & 87,0 & 8,7 & -- & -- & 17 & 11,5 & 36,5 & 46,2 & 5,8 & -- & -- \\
\hline 6 & -- & 32,1 & 43,3 & 20,8 & 3,8 & -- & 57 & 12,7 & 40,0 & 40,0 & 7,3 & -- & -- \\
\hline 23 & -- & 16,7 & 73,3 & 10,0 & -- & -- & 47 & -- & 57,6 & 42,4 & -- & -- & -- \\
\hline 50 & -- & 34,9 & 37,2 & 25,6 & 2,3 & -- & 45 & 13,5 & 38,5 & 44,2 & 3,8 & -- & -- \\
\hline 63 & -- & 29,2 & 52,0 & 18,8 & -- & -- & 42 & 8,9 & 46,7 & 40,0 & 4,4 & -- & -- \\
\hline 36 & -- & 18,4 & 73,7 & 7,9 & -- & - & 3 & 8,5 & 54,2 & 33,9 & 3,4 & -- & -- \\
\hline 43 & -- & 30,0 & 60,0 & 4,0 & 6,0 & -- & 2 & 36,2 & 43,1 & 15,5 & 5,2 & -- & -- \\
\hline
\end{tabular}

( ${ }^{1}$ ) Os tratamentos foram ordenados com base na nota média do índice de galhas e/ ou massas de ovos de modo decrescente.

$\left({ }^{2}\right)$ Três traços (--) indicam ausência de plantas com o respectivo índice de galhas e/ou massas de ovos. 
Neste trabalho, em nenhum genótipo ocorreu imunidade para $M$. paranaensis. O mesmo foi observado por MATA et al. (2002) e SERA et al. (2005) que não observaram imunidade para $M$. paranaensis, respectivamente, em C. arabica e em C. canephora. FAZUOLI et al. (1987) identificaram plantas da cultivar porta-enxerto Apoatã IAC-2258 de C. canephora com resistência a $M$. incognita, em condições de campo, em várias localidades do Estado de São Paulo, entretanto, GonçALVES et al. (1998) relatam que essa cultivar não é imune.

As 24 progênies da cultivar IPR-100 classificadas como resistentes são bastantes promissoras como novas cultivares de C. arabica. Propagadas por sementes, predominantemente, por autofecundação segundo Carvalho (1988), a cultivar pé franco IPR-100 de C. arabica é de custo mais baixo para a sua multiplicação do que a cultivar portaenxerto Apoatã IAC-2258 de C. canephora que necessita de enxertia.

A cultivar IPR-100 tem frutos de tamanho médio, semelhante ao Catuaí (SERA et al., 2002), mas há plantas de frutos menores juntamente com as de frutos maiores devido à segregação, tornando-se problemas no momento da colheita e comercialização. As plantas-mãe das progênies avaliadas neste trabalho são de frutos maiores em relação à cultivar IPR 100 original. Assim, essas 24 progênies comm resistência ao $M$. paranaensis têm potencial para se tornarem uma versão melhorada da cultivar IPR-100 com frutos maiores.

\section{AGRADECIMENTOS}

Os autores agradecem ao Consórcio Brasileiro de Pesquisa e Desenvolvimento do Café e ao Instituto Agronômico do Paraná por financiarem este trabalho.

\section{REFERÊNCIAS BIBLIOGRÁFICAS}

CARNEIRO, R. G. Reação de café 'Icatu' a Meloidogyne incognita raça 2 em condições de campo. Nematologia Brasileira, Brasília, v.19, n.1-2, p. 53-59, 1995.

CARNEIRO, R. M. D. G.; ALMEIDA, M. R. A. Distribution of Meloidogyne spp. on Coffee in Brazil: identification, characterization and intraspecific variability. In: MEJORAMIENTO SOSTENIBLE DEL CAFÉ ARABICA POR LOS RECURSOS GENÉTICOS, ASISTIDO POR LOS MARCADORES MOLECULARES, COM ÉNFASIS EN LA RESISTENCIA A LOS NEMÁTODOS, 2000, Turrialba. Publicación Especial. CATIE / IRD, Turrialba, 2000. p. 43-48.
CARVALHO, A. Principles and practice of coffee plant breeding for productivity and quality factors. Coffea arabica. In: CLARK, R. J.; MACRAE, R. (Ed.). Coffee: Agronomy. v. 4, London, 1988. p. 129- 165.

CRUZ, C. D. Programa Genes: versão Windows; aplicativo computacional em genética e estatística. Viçosa: UFV, 2001. 648p.

FAZUOLI, L.C.; COSTA, W.M.; GONÇALVES, W.; LIMA, M.M.A. Café Icatu como fonte de resistência e/ou tolerância ao nematóide Meloidogyne incognita. In: CONGRESSO BRASILEIRO DE PESQUISAS CAFEEIRAS, 11., 1984, Londrina. Resumos ... Rio de Janeiro: MIC/IBC, 1984. p.247-248.

FAZUOLI, L. C.; LIMA, M. M. A.; GONÇALVES, W.; COSTA, W. M.. Melhoramento do cafeeiro visando resistência a nematóides: utilização de porta-enxertos resistentes. In: CONGRESSO PAULISTA DE AGRONOMIA, 6, Piracicaba, 1987. Anais ... São Paulo: AEASP, 1987. p. 171-180.

GONÇALVES, W.; FERRAZ, L. C. C. B.; LIMA, M. M. A. de; SILVAROLLA, M. B. Reações de cafeeiros às raças 1, 2 e 3 de Meloidogyne incognita. Summa Phytopathologica, Jaguariúna, v. 22, n. 2, p. 172-177, 1996.

GONÇALVES, W.; LIMA, M. M. A. de; FAZUOLI, L. C. Resistência do cafeeiro a nematóides: III. Avaliação da resistência de espécies de Coffea e de híbridos interespecíficos a Meloidogyne incognita raça 3. Nematologia Brasileira, Brasília, v. 12 , p. $47-54,1988$.

GONÇALVES, W.; RAMIRO, D. A.; GALLO, P. B.; GIOMO, G. S. Manejo de nematóides na cultura do cafeeiro. In: REUNIÃO ITINERANTE DE FITOSSANIDADE DO INSTITUTO BIOLÓGICO - CAFÉ, 10., 2004, Mococa. Anais ... São Paulo: Instituto Biológico, 2004. p. 48-66.

GONÇALVES, W.; SILVAROLLA, M. B. Nematóides parasitos do cafeeiro. In: ZAMBOLIM, L. (Ed.). Tecnologias de produção de café com qualidade. Viçosa: UFV, Departamento de Fitopatologia, 2001. cap. 7. p. 199 - 268.

GONÇALVES, W.; SILVAROLLA, M. B.; LIMA, M. M. A. de. Estratégias visando a implementação do manejo integrado dos nematóides parasitos do cafeeiro. Informe Agropecuário, Belo Horizonte, v. 19, n. 193, p. 36-47, 1998.

KRZYZANOWSKI, A. A.; FIGUEREDO, R.; SANTIAGO, D. C.; FAVORETO, L. Levantamento de espécies e raças de Meloidogyne em cafeeiros no Estado do Paraná. In: SIMPÓSIO DE PESQUISA DOS CAFÉS DO BRASIL, 2, 2001, Vitória. Resumos..., Brasília: EMBRAPA Café, 2001. p. 81.

LORDELLO, A. I. L.; LORDELLO, R. R. A. Nematóides encontrados em cafezais do Estado de São Paulo. In: CONGRESSO BRASILEIRO DE NEMATOLOGIA, 23, 2001, Garça. Resumos ... Garça: SBN/ FAEF, 2001. p. 85.

MATA, J. S. da; SERA, T.; ALTÉIA, M. Z.; AZEVEDO, J. A.; FADELLI, S.; PETEK, M. R.; TRILLER, C.; SERA, G. H. Resistência de genótipos de cafeeiro (Coffea arabica L.) de São Jorge do Patrocínio ao nematóide Meloidogyne paranaensis (EMN2001.07). SBPN - Scientific Journal (Ed. Especial), São Paulo, v. 6, p. 34 - 36, 2002. 
MATA, J. S. da; SERA, T.; AZEVEDO, J. A.; ALTÉIA, M. Z.; COLOMBO, L. A.; SANCHES, R. S.; PETEK, M. R.; FADELLI, S. Seleção para resistência ao nematóide Meloidogyne paranaensis EMN-95001: IAPARLN 94066 de "Catuaí x Icatu" em área altamente infestada. In: SIMPÓSIO DE PESQUISA DOS CAFÉS DO BRASIL, 1, 2000, Poços de Caldas. Resumos expandidos. Brasília: EMBRAPA, 2000. p. 515 - 518.

SANTOS, J. M. dos. Estudo das principais espécies de Meloidogyne goeldi que infectam o cafeeiro no Brasil com descrição de Meloidogyne goeldii sp. n. 1997. 153 f. Tese (Doutorado). Botucatu: UNESP/FCA.

SANTOS, J. M.. Os nematóides de galha que infectam o cafeeiro no Brasil. In: REUNIÃO ITINERANTE DE FITOSSANIDADE DO INSTITUTO BIOLÓGICO, 4. In: Encontro sobre doenças e pragas do cafeeiro, 5, 2001, Ribeirão Preto. Anais ... Ribeirão Preto: Instituto Biológico, 2001. p. 10-20.

SASSER, J. N. Plant-parasitic nematodes: the farmer's hidden enemy. Raleigh: North Caroline State University Graphics, 1979. 115 p.

SASSER, J. N.; CARTER, C. C.; HARTMAN, K. M. Standardization of host suitability studies and reporting of resistence to root-knot nematodes. Raleigh: North Caroline State University Graphics, 1984. 7 p.

SERA, T.; ALTÉIA, M. Z.; PETEK, M. R.; MATA, J. S. da. Novas cultivares para o modelo IAPAR de café adensado para o Paraná. In: CONGRESSO BRASILEIRO DE PESQUISAS CAFEEIRAS, 28, 2002, Caxambu. Trabalhos apresentados... Rio de Janeiro: MAPA/PROCAFÉ, 2002. p. 432 - 434.
SERA, T.; MATA, J. S.; ITO, D. S.; DOI, D. S.; SERA, G. H.; AZEVEDO, J. A.; COTARELLI, V. M. Identificação de cafeeiros resistentes aos nematóides Meloidogyne paranaensis e $M$. incognita raças 2 e 1 em populações de Icatu (Coffea arabica). SBPN Scientific Journal, São Paulo, v. 8, p. 20, 2004a.

SERA, T.; MATA, J. S. da; SERA, G. H.; DOI, D. S.; ITO, D. S.; AZEVEDO, J. A. de; COTARELLI, V. M. Freqüência de plantas resistentes aos nematóides Meloidogyne paranaensis e $M$. incognita raças 2 e 1 em populações da cultivar porta-enxerto Apoatã de Coffea canephora. SBPN Scientific Journal, São Paulo, v. 8, p. 17, 2004b.

SERA, T.; MATA, J. S. da; SERA, G. H.; DOI, D. S.; ITO, D. S.; AZEVEDO, J. A. de; RIBEIRO-FILHO, C. Identificação de porta-enxertos de café Robusta resistentes aos nematóides $M$. paranaensis e $M$. incognita raças 2 e 1. In: SIMPÓSIO DE PESQUISA DOS CAFÉS DO BRASIL, 4., 2005, Londrina. Anais... Londrina, 2005. CD-Rom

TAYLOR, A. L. Introduction to research on plant nematology, an FAO guide to study and control of plant parasitic nematodes. Rome: FAO, UN, 1971. PL:CP/5-rev.1.

TAYLOR, A. L.; SASSER, J. N. Biology: identification and control of root-knot nematodes (Meloidogyne species). Raleigh, USA: NCSU \& USAID, 1978. 111p. 